\title{
Temporal structure of diving behaviour in sympatric Antarctic and subantarctic fur seals
}

\author{
Sebastián P. Luque ${ }^{1,2, *}$, John P. Y. Arnould ${ }^{3}$, Christophe Guinet ${ }^{2}$ \\ ${ }^{1}$ Department of Biology, Memorial University, St. John's, Newfoundland and Labrador A1B 3X9, Canada \\ ${ }^{2}$ Centre d'Etudes Biologiques de Chizé, CNRS, 79360 Villiers en Bois, France \\ ${ }^{3}$ School of Life and Environmental Sciences, Deakin University, 221 Burwood Highway, Burwood, Victoria 3125, Australia
}

\begin{abstract}
Lactation is considerably briefer (4 vs. $10 \mathrm{mo}$ ) and daily pup energy expenditure higher in Antarctic (AFS) than in subantarctic fur seals (SFS), even in sympatric populations of both species, where their foraging locations and diets are similar. Therefore, lactational demands may be higher for AFS females. We investigated whether sympatric lactating AFS and SFS females differ in their physiological or behavioural diving capacities, and in the temporal structure of foraging behaviour. Mean dive depth and duration were greater in SFS, but dives below $140 \mathrm{~m}$ were performed only by AFS. An index of activity level during the bottom phase of dives, when fur seals are thought to capture prey, was higher in SFS. Despite these differences, SFS females showed a steady increase in the minimum postdive interval following dives lasting longer than $250 \mathrm{~s}$, compared to the steady increase following dives lasting longer than only $150 \mathrm{~s}$ in AFS. These results suggest that physiological constraints on diving behaviour are stronger on AFS females, and that the behavioural aerobic dive limit is greater for SFS. Assuming that dive bouts reflect foraging in prey patches, AFS females exploited more patches per unit time, and remained in them for briefer periods of time, compared to SFS females. Dive bout structure did not differ between overnight and long foraging trips. Our data suggest that AFS females spend greater foraging effort, but may gain access to prey patches of better quality, which may help them cope with higher lactational demands.
\end{abstract}

KEY WORDS: Optimal foraging · Foraging niche segregation · Foraging behaviour · Diel trends · Diurnal activity $\cdot$ Myctophidae $\cdot$ Syntopy $\cdot$ Archival tags

\section{INTRODUCTION}

Exploitative competition for food between animals is difficult to assess, partly because it requires knowledge of whether the level of their common resources is limiting or not (Birch 1957, MacArthur \& Levins 1964). Yet it is considered a major component of species interactions, and influences community structure (Milinski \& Parker 1991). In the absence of data on the level of common resources, exploitative competition is often inferred from measurements of resource overlap or manipulations of population densities (Schoener 1974). However, an approach based on comparisons of the biology and behaviour of potentially competing species may be more useful for understanding inter- specific interactions (Tilman 1987). This approach has been used to study mechanisms of coexistence in a broad range of species (Stamps 1983, Cowlishaw 1999, Hull 1999, Robinson et al. 2002).

Using this approach, Ballance et al. (1997) showed that competitive ability and energetic constraints play a major role in the structure of seabird assemblages. Sympatry or syntopy in morphologically similar, related species is particularly attractive for studying these interactions. Under such conditions, it is possible to compare the ecology of co-occurring species, while minimizing the influence of extraneous factors, which are difficult or impossible to control. Studies of related seabirds have provided evidence of physiological constraints on the behavioural response of coexisting 
predators to heterogeneity in the distribution of common prey resources (Hull 1999, Mori \& Boyd 2004b). Therefore, studies such as these can be used as a basis for predicting the outcome of reductions in prey abundance and/or availability.

Lactation constrains the foraging behaviour of mammals (Sæther \& Gordon 1994), especially in the marine environment (Costa \& Williams 1999). Therefore, new insights into the mechanisms linking prey distribution and predator behaviour can be gained by comparing the foraging behaviour of sympatric marine mammals. Otariid (fur seals and sea lions) pinnipeds are good models in comparative foraging ecology because they exhibit large variation in adult body size and lactation duration, while other life history traits are relatively homogeneous (Ridgway \& Harrison 1981). Female body mass varies from 30 (in Galápagos fur seals Arctocephalus galapagoensis [Heller 1904]) to $275 \mathrm{~kg}$ (in Steller sea lions Eumetopias jubatus [Schreber 1776]), and lactation duration varies from 4 (in Antarctic fur seal Arctocephalus gazella [Peters 1875]) to 36 mo (in Galápagos fur seals; range 12 to $36 \mathrm{mo}$; Trillmich \& Kooyman 1986). Large pinnipeds have higher aerobic dive limits (ADL) than smaller ones (Kooyman 1989), so they can dive for longer periods of time (Halsey et al. 2006) without the negative effects of increased lactic acid concentration. Consequently, they have more time available for prey search and capture. However, species of similar body mass can display large differences in lactation duration, energy transfer to pups, and patterns of energy allocation by pups (Arnould 1997, Arnould et al. 2003). Therefore, the interplay among body mass, foraging behaviour, and lactation duration remain unclear. Sympatric otariids of similar body mass, yet different lactation duration, offer a unique opportunity to understand the physiological and behavioural tradeoffs faced by breeding females during lactation.

Diving marine predators often dive in bouts, i.e. most dives occur in rapid succession, while the rest are separated by longer intervals, and each bout may indicate foraging in a particular patch (Mori \& Boyd 2004a). Researchers thus deduced that the temporal structure of diving may be used to infer the distribution and characteristics of foraging patches in several seabirds (Kato et al. 2000, Mehlum et al. 2001), and pinnipeds (Mori \& Boyd 2004a, Mori et al. 2005). This provides a framework for comparing the foraging behaviour of sympatric marine mammals in relation to a common prey resource.

Antarctic and subantarctic (A. tropicalis [Gray 1872]) fur seals (AFS and SFS, respectively, hereafter) have broadly different spatial distributions. AFS breed mostly south of the Antarctic Polar Front, while SFS do so mostly north of it, but they breed sympatrically at 3 locations (Bonner 1999): Macquarie Island, Ile de la Possession (Iles Crozet), and Marion Island (Prince Edward Islands). Adult females of these species have similar body mass and show little differences in at-sea distribution and diet when sympatric (Klages \& Bester 1998, Robinson et al. 2002, Bailleul et al. 2005, Luque et al. 2007a), yet wean pups at 4 in (AFS) vs. 10 mo of age in (SFS) (Bester \& Bartlett 1990), as they do in allopatric populations. Furthermore, AFS pups show higher daily energy expenditures (Arnould et al. 2003), and grow in body length faster (Luque et al. 2007b) at Ile de la Possession. Thus, their mothers must meet these demands in addition to their own, and also complete lactation in $<\frac{1}{2}$ the time compared to SFS. Lactational demands may thus be higher in AFS, which females should satisfy during their foraging trips to sea.

Previous comparisons of diving behaviour between these species in sympatry have not shown major differences at the scale of entire foraging trips (Goldsworthy et al. 1997, Robinson et al. 2002). At Ile de la Possession, however, overnight foraging trips (OFTs) are significantly more frequent in SFS, and AFS appear to spend a greater proportion of their time at sea (Bester \& Bartlett 1990, Bailleul et al. 2005, Luque et al. 2007a), as in Marion Island. Differences at finer scales have been studied at Ile de la Possession, where lactating female AFS exhibit stronger diel variation in dive depth, with relatively shallow diving (AFS: 25 to $30 \mathrm{~m}$; SFS: 35 to $50 \mathrm{~m}$ ) during the noncrepuscular hours of the night, and deep diving (AFS: 45 to $65 \mathrm{~m}$; SFS: 40 to $50 \mathrm{~m}$ ) during crepuscular hours (Luque et al. 2007a). At Marion (Klages \& Bester 1998, Ferreira \& Bester 1999) and Macquarie Islands (Robinson et al. 2002), no interspecific differences in diet were found. Scat analyses suggest that sympatric AFS and SFS feed on the same prey species (myctophid fish), although differences in their relative abundance were found at Ile de la Possession (Luque et al. 2007a). Comparisons of the temporal structure of diving behaviour between AFS and SFS, however, are unavailable, but may help us understand how these species with different lactational demands respond to distribution of similar prey.

Diving capacity of otariid species with similar body mass is expected to be similar (Kooyman 1989), assuming other factors do not differ. In this case, optimal diving models predict similar optimal foraging depth (Mori 1998a, 2002). Optimal diving models typically assume that divers maximize energy intake rate, although predation risk may significantly influence such predictions (Frid et al. 2007). They also predict that divers should increase the time spent in foraging patches as depth increases (Mori 1998b, Thompson \& Fedak 2001), but only if diving remains aerobic (Houston \& Carbone 1992) or patch quality (as reflected by 
prey density) increases. A previous study does not support the first prediction of similar optimal foraging depth (Luque et al. 2007a), suggesting that important physiological or behavioural differences exist between female AFS and SFS, which may affect their exploitation of prey patches. Therefore, we investigated whether the temporal structure of diving behaviour and vertical distribution of foraging patches differ between fur seal species at Ile de la Possession. Given the briefer lactation and higher daily energy requirements of AFS pups, we tested the hypothesis that female AFS have higher energy demands during lactation, and should spend more foraging effort, despite their overlapping foraging areas.

\section{MATERIALS AND METHODS}

Data on diving behaviour of AFS and SFS was obtained at La Mare aux Elephants $\left(46^{\circ} 22^{\prime} 29^{\prime \prime} \mathrm{S}\right.$, $\left.51^{\circ} 40^{\prime} 13^{\prime \prime} \mathrm{E}\right)$, at the western end of Ile de la Possession, Crozet archipelago, Southern Indian Ocean, during the 2001-02 (4 December to 25 March) and 2002-03 (1 December to 16 March) breeding seasons (2001 and 2002 hereafter). A total of 277 (AFS: 153, SFS: 124) pups were individually marked as previously described (Georges \& Guinet 2000, Arnould et al. 2003, Bailleul et al. 2005), providing a means to identify their mothers, a subset of which were equipped with time and depth recorders (TDRs; Table 1).

Diving behaviour. Animal capture and handling procedures are described in Luque et al. (2007a). Briefly, lactating females of each species were captured on land during their nursing visits. Each individual was weighed to the nearest $\mathrm{kg}$, and placed on a restraint board for attachment of instruments. One of 3 different time-depth recorder (TDR) models (MK5, MK7, and MK8; Wildlife Computers) was glued to the dorsal fur between the scapulae, using a 2-component epoxy glue (AW 2101 Ciba Specialty Chemicals). Fur seals trav- elled 50 to $100 \mathrm{~km}$ from the colony to forage, and there were no significant differences in foraging trip duration, dive depth, or dive duration between individuals instrumented with different TDR models $(p>0.5$ in all cases). Thus, data from the 3 models were pooled for interspecific comparisons. TDRs were programmed to record time and depth every $5 \mathrm{~s}$ when the seals were at sea, with a $1 \mathrm{~m}$ depth resolution. MK8 TDRs also recorded speed to the nearest $0.02 \mathrm{~m} \mathrm{~s}^{-1}$. Instruments were left on the seals for 1 to 11 foraging trips, and were recovered by cutting the fur beneath them, upon the seals' return to the colony.

TDR data were downloaded to a portable computer, and analyses of the resulting time series of dive data were performed using custom written software package diveMove (Luque 2007), available through GNU R (R Development Core Team 2007). Before analyses, depth readings were corrected for shifts in the pressure transducer of the TDR. Sections of each record were identified as foraging trips if continuous wet activity (i.e. continuous depth readings) was available for at least 6 h. This limit was selected to exclude brief excursions to sea for activities other than foraging, which occurred mostly during daytime, and contained isolated shallow dives $(<20 \mathrm{~m})$, in contrast to the bout-organized dives typical of foraging trips (Luque et al. 2007a).

Dives were defined as departures from the surface to depths $\geq 4 \mathrm{~m}$ plus the ensuing return to the surface. Dives to lower depths were not considered, as they were indistinguishable from noise remaining after adjustment of pressure transducer drifts, which was greater than the resolution of the instrument (Beck et al. 2000). Each dive was divided into descent, bottom, and ascent phases, where: (1) descent started at the surface and ended when no further increases in depth were detected; (2) ascent was defined from the end of the dive and, with the reversed time series, ending when no further increases in depth were detected; and (3) the bottom phase was the period between descent and ascent. Dives were thus described by their

Table 1. Arctocephalus gazella (AFS), A. tropicalis (SFS). Summary of dive data obtained from lactating females fitted with timedepth recorders (TDRs) on Ile de la Possession, in the breeding seasons of 2001-02 and 2002-03; BEC = bout-ending criterion. Data are either number or mean $\pm \mathrm{SE}$

\begin{tabular}{|lcccccccccc|}
\hline Species & $\begin{array}{c}\text { Breeding } \\
\text { season }\end{array}$ & $\begin{array}{c}\text { Body mass } \\
(\mathrm{kg})\end{array}$ & $\mathrm{N}$ & $\begin{array}{c}\text { Foraging } \\
\text { trips }\end{array}$ & Dives & BEC (s) & Bouts & $\begin{array}{c}\text { Dives in } \\
\text { bouts }\end{array}$ & $\begin{array}{c}\text { Mean dive } \\
\text { depth (m) }\end{array}$ & $\begin{array}{c}\text { Mean dive } \\
\text { duration }(\mathrm{s})\end{array}$ \\
\hline AFS & 2001 & $33.2 \pm 0.44$ & 37 & 102 & 42095 & $54.9 \pm 0.44$ & 3814 & 33522 & $31.2 \pm 0.32$ & $80.1 \pm 0.60$ \\
& 2002 & $31.6 \pm 0.58$ & 12 & 30 & 17541 & $38.5 \pm 0.83$ & 1614 & 13500 & $22.8 \pm 0.70$ & $73.4 \pm 1.32$ \\
& Both & $32.7 \pm 0.36$ & 49 & 132 & 59636 & $50.9 \pm 0.33$ & 5428 & 47022 & $29.2 \pm 0.24$ & $78.5 \pm 0.42$ \\
SFS & & & & & & & & & & \\
& 2001 & $30.6 \pm 0.55$ & 37 & 113 & 39102 & $84.8 \pm 0.91$ & 2839 & 33780 & $42.1 \pm 0.44$ & $94.2 \pm 0.63$ \\
& 2002 & $30.4 \pm 0.78$ & 10 & 32 & 17693 & $72.8 \pm 1.44$ & 1156 & 15302 & $32.9 \pm 0.70$ & $90.2 \pm 1.68$ \\
& Both & $30.5 \pm 0.45$ & 47 & 145 & 56795 & $81.7 \pm 0.61$ & 3995 & 49082 & $39.7 \pm 0.31$ & $93.2 \pm 0.46$ \\
\hline
\end{tabular}


duration, maximum depth, bottom time, and postdive duration.

Fur seals catch their prey predominantly during the bottom phase of dives, although this has been demonstrated only for AFS (Hooker et al. 2002). As a measure of the level of activity during the bottom (foraging) phase of dives, we thus divided the absolute number of vertical meters each individual swam during this phase by bottom time. We also calculated mean swimming speed during the bottom phase of dives from fur seals deployed with MK8 TDRs. Speed measurements, however, do not correspond to true speed because they need to be calibrated against the true speed of the seals. Nonetheless, we used measured speed because we were more interested in comparing the levels of activity during the bottom phase of dives between species, rather than estimating true speed.

To investigate whether physiological constraints differ between species, we studied the relationship between the briefest postdive duration and dive duration binned at $5 \mathrm{~s}$ intervals. The dive duration beyond which the briefest postdive interval begins to rise was taken as a behavioural proxy for the ADL, i.e. the dive duration beyond which blood lactate levels rise, as a result of increasing anaerobic metabolism (Kooyman 1989).

Identification of dive bouts. Identification of bouts of most behaviours has traditionally relied on log-survivorship or log-frequency analysis (Gentry \& Kooyman 1986, Sibly et al. 1990). However, Langton et al. (1995) pointed out that these methods include some level of subjectivity. The procedure involves fitting a curve to histogram data, an arbitrary choice of histogram class width, and adjustment for empty class intervals. Langton et al. (1995) presented an improved approach, based on maximum likelihood estimation, which uses the entire dataset to describe the frequency distribution of events such as diving. This procedure was used to model the distribution of sequential differences in surface interval duration, allowing for variation in dive characteristics between bouts (Mori et al. 2001). The $\log$ likelihood $\left(\log L_{2}\right)$ of all $N_{t}$ absolute differences in surface interval duration $t$ is a combination of fast (within bouts) and slow (between bouts) events (Luque \& Guinet 2007):

$$
\log L_{2}=\sum_{i=1}^{N_{t}} \log \left[p \lambda_{\mathrm{f}} \mathrm{e}^{-\lambda_{\mathrm{f}} t_{\mathrm{i}}}+(1-p) \lambda_{\mathrm{s}} \mathrm{e}^{-\lambda_{\mathrm{s}} t_{\mathrm{i}}}\right]
$$

where $p$ is a mixing parameter representing the proportion of fast to slow process events in the sampled population; the subscripts $\mathrm{f}$ and $\mathrm{s}$ denote the fast and slow processes, respectively, and $\lambda$ represents the probability of an event occurring in a given process per unit time $t$ (Sibly et al. 1990). A bout ending criterion (BEC), determining whether two successive dives should be grouped in the same bout or not, can be calculated from Eq. (1) as:

$$
\mathrm{BEC}=\frac{1}{\lambda_{\mathrm{f}}-\lambda_{\mathrm{s}}} \log \frac{p \lambda_{\mathrm{f}}}{(1-p) \lambda_{\mathrm{s}}}
$$

If the difference in surface interval duration between two successive dives exceeded the $\mathrm{BEC}$, then they were grouped in different bouts.

Statistical approach. BECs were separately determined for each individual due to individual differences in foraging trip durations, and to avoid masking of bout structure by individual differences in diving behaviour. The number of bouts, their duration, and the duration of intervals between bouts were also similarly calculated. Because both AFS and SFS dived almost exclusively at night, the first two variables were divided by the number of night hours available during the foraging trip of each individual. Night time was defined as the number of hours between sunset and sunrise. Bouts consisting of a single dive were not deemed to indicate foraging behaviour, and were thus excluded from analyses.

Both AFS and SFS displayed bimodal distributions of foraging trip duration (Luque et al. 2007a), with overnight $(\mathrm{OFT},<1 \mathrm{~d})$ and long foraging trips (LFT, $>1 \mathrm{~d})$. Therefore, interspecific comparisons included fixed effects terms to test for differences between these types of foraging trip. Data for multiple trips of the same type were averaged for each individual, except for bout and postbout duration, which represent variation within the foraging trip. A single value per individual and bout was considered for bout and postbout duration. A random effect term for individuals was included, so mixed effects models (Pinheiro \& Bates 2000) were used to describe data for the ith individual, $j$ th species, and $k$ th foraging trip type, as follows:

$$
\begin{gathered}
y_{i j k}=\beta_{0}+\beta_{j}+\beta_{k}+\beta_{j k}+b_{i}+\varepsilon_{i j k} \\
b_{i} \sim \mathcal{N}\left(0, \sigma_{b}^{2}\right), \varepsilon_{i j k} \sim \mathcal{N}\left(0, \sigma^{2}\right)
\end{gathered}
$$

where $\beta_{0}$ represents an intercept; $\beta_{j}, \beta_{k}, \beta_{j k}$ represent the fixed effects for species, foraging trip type, and corresponding interaction, respectively; $b_{i}$ denotes the random effect for individual, and $\varepsilon_{i j k}$ denotes an independent error term. The $b_{i}$ and $\varepsilon_{i j k}$ terms are assumed to be normally distributed, with mean zero, and variances $\sigma_{b}^{2}$ and $\sigma^{2}$, respectively. Multiple bout and postbout durations for the same individual were, thus, included as part of $\varepsilon_{i j k}$ for the corresponding models.

The significance of the interactions was assessed by comparing the models with and without the interaction term, using a log likelihood ratio test (Pinheiro \& Bates 2000). The significance of other fixed effects was tested via Markov Chain Monte Carlo (MCMC) simulations based on samples of the posterior distribution of the 
model parameters (Baayen et al. 2008). All analyses were carried out in the GNU R system (R Development Core Team 2007), with packages diveMove for dive behaviour analysis (Luque 2007) and lme 4 for fitting mixed effects models (Bates et al. 2008). Results are presented as means $\pm \mathrm{SE}$, unless stated otherwise.

\section{RESULTS}

Dive data from a total of 96 females were obtained, providing information from 277 foraging trips (Table 1). Most dives ( $83 \%$ ) occurred in bouts for both species. However, this proportion was significantly larger $\left(\chi^{2}=1157, \mathrm{df}=1, \mathrm{p}<0.001\right)$ in SFS (86.4 vs. $\left.78.8 \%\right)$, indicating that isolated dives were relatively more common in AFS. The number of bouts per night of foraging trip was larger in AFS (log-transformed to normalize residuals; $F_{1,148}=58.85, \mathrm{p}<0.001$ ) (Fig. 1a), regardless of foraging trip type (interaction term: $\chi^{2}=0.08, \mathrm{p}=$ $0.78)$. No significant differences between foraging trip types were found $\left(F_{1,148}=0.07, \mathrm{p}=0.8\right)$, and the pooled estimates for each species were $35.7 \pm 1.05$ and $21.5 \pm$ 1.05 bouts per night for AFS and SFS, respectively. Typical dive profiles are shown in Appendix 1 www.int-res.com/articles/suppl/m372p277_app.pdf.

The BEC was significantly higher for SFS during both seasons $\left(F_{1,88}=52.39, \mathrm{p}<0.001\right)$, and higher for both species during the 2001 season $\left(F_{1,88}=7.42, \mathrm{p}<\right.$ 0.01 ). Although this suggests that foraging conditions may have differed between seasons, data for both seasons were pooled for further analyses because there was no interaction between species and season $\left(F_{1,88}=\right.$ $1.55, p=0.2$ ), and interannual differences were not the focus of this paper.

\section{Dive bout structure}

The total time spent in bouts per night was similar between species $\left(F_{1,144}=2.00, \mathrm{p}=0.2\right)$ and foraging trip types $\left(F_{1,144}=0.01, \mathrm{p}=0.9\right)$, without any interaction between both factors (interaction term $\chi^{2}=0.01, p=0.92$ ) (Fig. 1b). Pooled estimates for each species were $9.6 \pm$ 1.05 and $10.6 \pm 1.05 \mathrm{~h} \mathrm{night}^{-1}$ for AFS and SFS, respectively. Bout duration, however, was significantly longer in SFS during both foraging trip types $\left(F_{1,9520}=43.37\right.$, $\mathrm{p}<0.001$ ) (Fig. 1c) consistent with the higher number of bouts per night for AFS, but similar time spent in those bouts. Bout duration was similar between foraging trip types $\left(F_{1,9520}=0.24, \mathrm{p}>0.9\right)$, independently of differences between species $\left(\chi^{2}=0.88, p=0.35\right)$.

The temporal distribution of foraging patches, as reflected by the density distribution of postbout durations, showed that AFS encountered patches at a
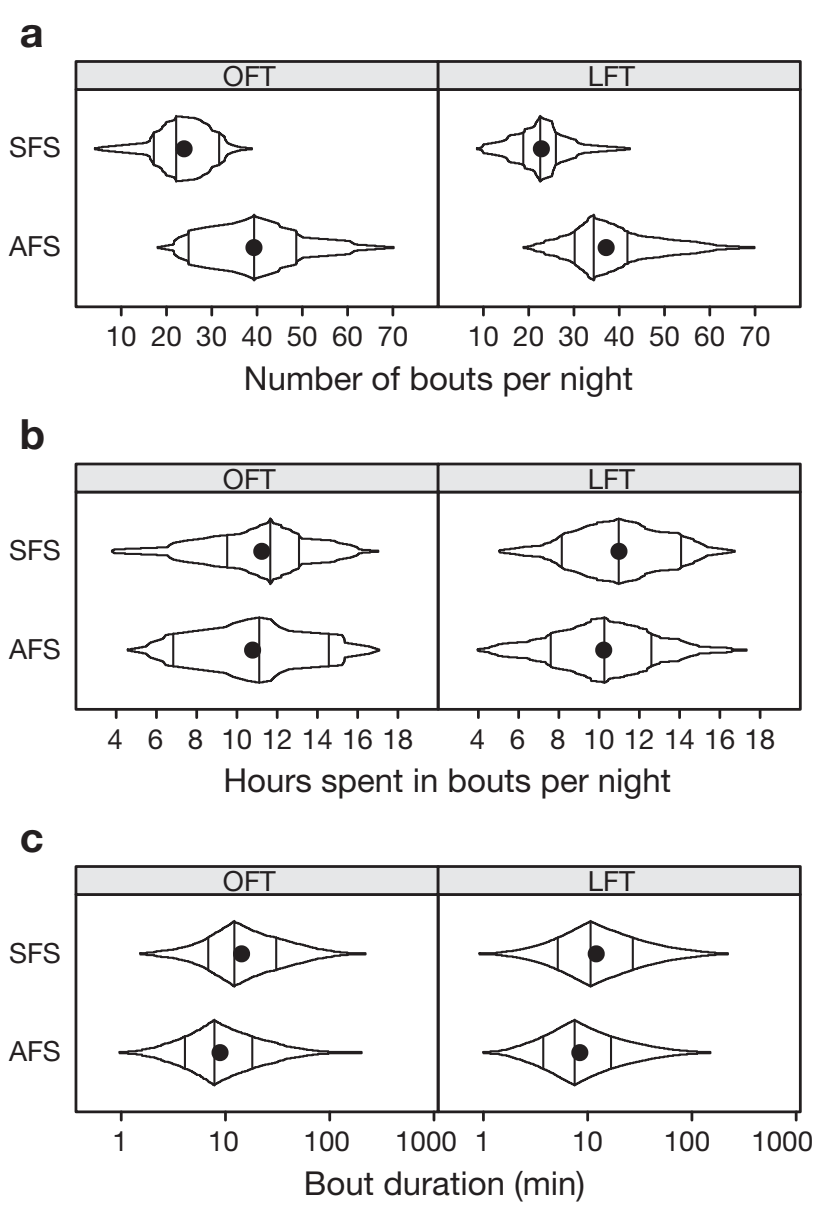

Fig. 1. Arctocephalus gazella (AFS), A. tropicalis (SFS). OFT: overnight foraging trips; LFT: long foraging trips. Lactating AFS females perform more bouts per night, spend a similar amount of time in bouts per night, and have briefer bouts than lactating SFS females

slightly faster rate than SFS (Fig. 2). Albeit small, the difference was significant for OFTs (Kruskal-Wallis $\left.\chi^{2}=4.60, \mathrm{df}=1, \mathrm{p}=0.03\right)$ and LFTs $\left(\chi^{2}=91.68, \mathrm{df}=1\right.$, $p<0.001)$. Patch encounter rate, as measured by postbout duration, did not differ significantly between foraging trip types $\left(\chi^{2}=2.62, \mathrm{df}=1, \mathrm{p}=0.11\right)$. Foraging bouts were most frequent near the surface for both species, but their relative frequencies were higher near the surface and below $80 \mathrm{~m}$ in AFS, while depths between 30 and $60 \mathrm{~m}$ were more heavily used by SFS females (Fig. 3).

\section{Behavioural and physiological limits}

The absolute number of meters that fur seals swam per unit time during the bottom phase of dives was significantly higher in SFS $\left(F_{1,149}=13.2, \mathrm{p}<0.001\right)$, and 


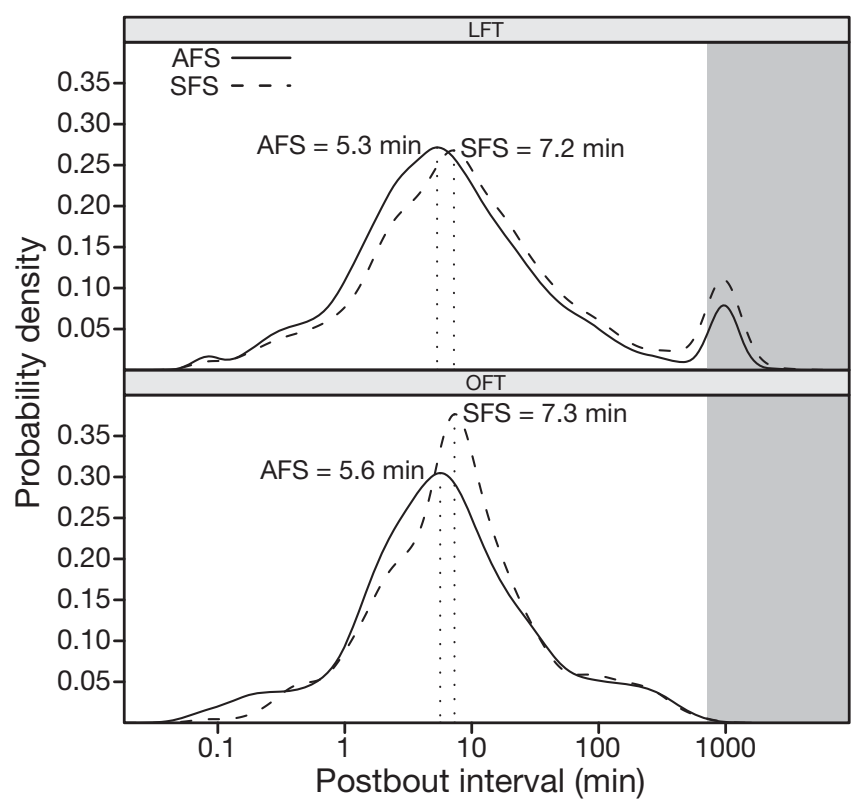

Fig. 2. Arctocephalus gazella (AFS), A. tropicalis (SFS). Kernel density estimates of postbout intervals during overnight foraging trips (OFT) and long foraging trips (LFT). The temporal separation between foraging patches, as indicated by postbout intervals, is briefer in lactating Antarctic (AFS) than in subantarctic fur seal (SFS) females, during both LFT and OFT. Dotted lines indicate the postbout interval with the highest kernel density estimate. Shaded regions denote intervals $>12 \mathrm{~h}$, i.e. bouts occurring on different foraging nights

was independent of foraging trip type $\left(\chi^{2}=2.08, \mathrm{p}=\right.$ $0.15)$. SFS swam $0.13 \pm 0.006 \mathrm{~m} \mathrm{~s}^{-1}$, and AFS $0.10 \pm$ $0.006 \mathrm{~m} \mathrm{~s}^{-1}$ while at the bottom of dives. Furthermore, this rate was significantly higher $\left(F_{1,149}=22.3, \mathrm{p}<\right.$ $0.001)$ during LFTs $\left(0.12 \pm 0.005 \mathrm{~m} \mathrm{~s}^{-1}\right)$ than during OFTs $\left(0.10 \pm 0.006 \mathrm{~m} \mathrm{~s}^{-1}\right)$ for both fur seal species.

The mean swimming speed during the bottom phase of dives did not differ between species $\left(F_{1,58}<0.01, \mathrm{p}>\right.$ $0.9)$, nor between foraging trip types $\left(F_{1,58}=0.14, \mathrm{p}=\right.$ $0.7)$, and no interaction between these factors $\left(\chi^{2}=\right.$ $0.01, p=0.9$ ) was noted. Similarly, mean swimming speed during surface intervals did not differ between species for OFTs $\left(F_{1,20}=1.18, \mathrm{p}=0.29\right)$, nor for LFTs $\left(F_{1,36}=4.1, \mathrm{p}=0.05\right)$, despite a significant interaction between foraging trip type and species $\left(\chi^{2}=4.35, \mathrm{p}=\right.$ $0.04)$

The relationship between the duration of the briefest surface interval and dive duration showed no changes in surface interval for dives lasting up to almost $150 \mathrm{~s}$ in both species (Fig. 4). However, surface intervals following dives longer than $150 \mathrm{~s}$ increased steadily for AFS, whereas SFS showed a similar response only for dives longer than $250 \mathrm{~s}$ (Fig. 4), despite the latter's larger rate of vertical movements at the bottom of dives.

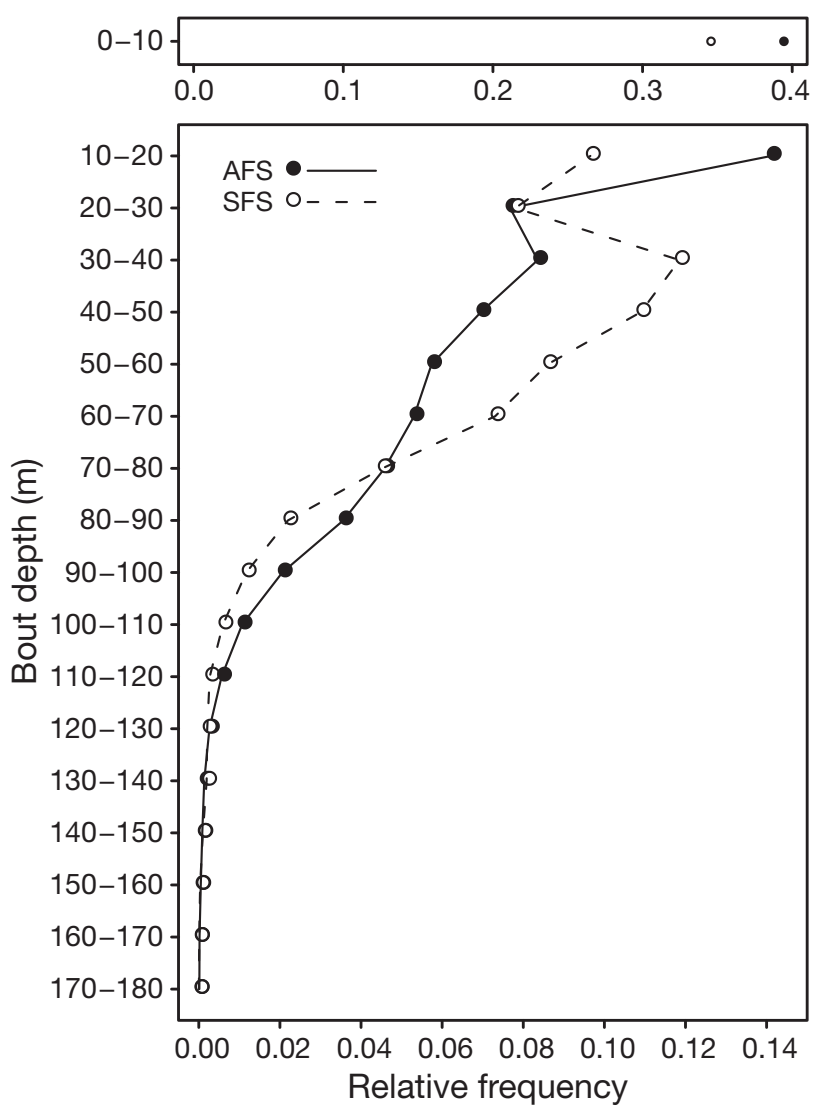

Fig. 3. Arctocephalus gazella (AFS), A. tropicalis (SFS). Lactating Antarctic fur seal (AFS) females forage more frequently than subantarctic (SFS) females between the surface and $20 \mathrm{~m}$, and below $80 \mathrm{~m}$. SFS females spend a greater proportion of their bouts between 30 and $60 \mathrm{~m}$

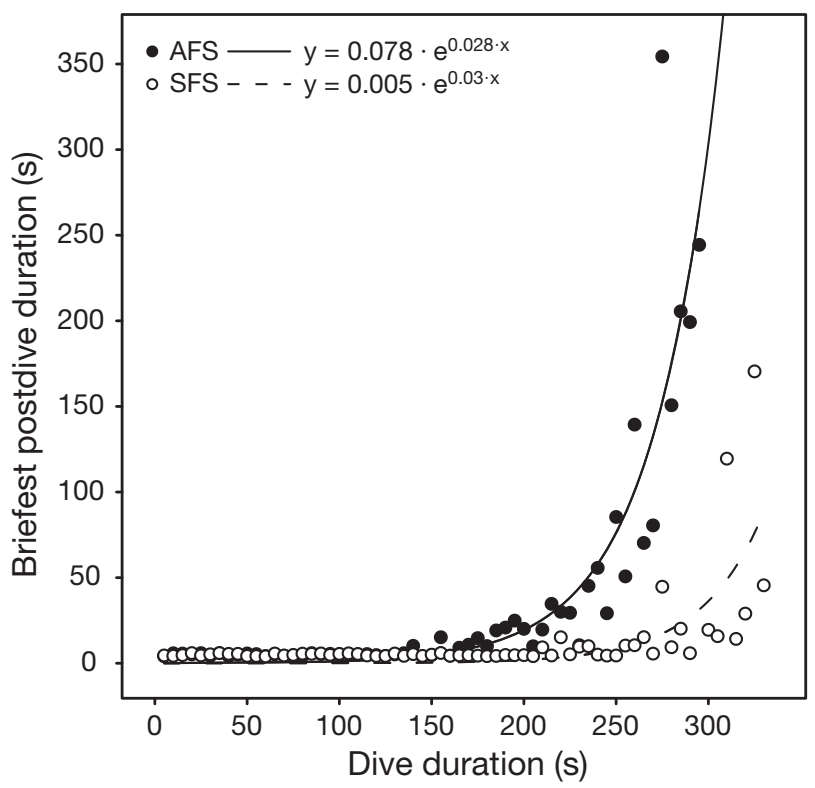

Fig. 4. Arctocephalus gazella (AFS), A. tropicalis (SFS). Briefest surface postdive intervals increase exponentially for dive durations longer than 150 and $250 \mathrm{~s}$ in lactating Antarctic (AFS) and subantarctic fur seal (SFS) females, respectively 


\section{DISCUSSION}

We hypothesized that lactating female AFS have higher energy demands during lactation and, therefore, should spend greater foraging effort to meet those demands. Female AFS displayed higher indices of diving effort, reduced patch residence times, and briefer postbout intervals, suggesting more effort spent foraging, and a faster rate of encounter with prey patches. An important premise of our analyses is that both species forage optimally, i.e. that they maximize energy intake rate relative to physiological constraints. Previous results have shown subtle interspecific differences in diet and dive depth associated with differences in life history and pup physiology (Luque et al. 2007a), so behavioural and physiological constraints may not be similar between AFS and SFS. Moreover, optimal foraging behaviour (e.g. time spent in foraging patches and patch encounter rate) may also differ between species, and affect their rates of energy intake. Our results largely support these predictions, and suggest a possible origin for the differences in foraging behaviour. We review the assumptions that were required to use our approach, and discuss the implications of our results for inferring the characteristics of the foraging habitat of sympatric AFS and SFS.

\section{Assumptions}

Using the information on differences in physiological constraints required making assumptions regarding the diving behaviour of fur seals (Mori et al. 2002, 2005, Mori \& Boyd 2004b). A major assumption of the model is that energy intake is a function of time spent at the bottom of dives, i.e. prey is assumed to be obtained during the bottom phase of dives. Although it was not possible to ascertain the validity of this assumption with a 2-dimensional dive profile and sampling interval of $5 \mathrm{~s}$, direction reversals (wiggles) during descent or ascent phases were rare. Furthermore, the mean vertical distance covered during the bottom phase of dives (a measure of the amount of wiggling) was relatively high for both species $(\mathrm{AFS}=12.7 \mathrm{~m}$; SFS $=15.0 \mathrm{~m}$ ) for dive depths $>10 \mathrm{~m}$. These values exclude shallow V-shaped dives, which are indicative of travelling behaviour. Therefore, fur seals from La Mare aux Elephants probably concentrated their energy intake during bottom time.

A more fundamental assumption of the model is that fur seals dived optimally, so as to maximize the rate of energy intake during the dive cycle. Foragers may not always use an energy intake rate maximizing currency to behave optimally (Caraco 1980), as other factors may play important roles in determining what behav- iour is optimal under particular time scales. Nonacs (2001) reviewed several studies testing predictions based on this assumption and found a consistent bias in the predictions, arguing that inclusion of the forager's state (e.g. nutritional status and predation risk) in the models can improve their predictive power. Heithaus \& Frid (2003) proposed a model to account for predation risk during the surface interval for diving predators, which may partly explain why diving behaviour is often considered suboptimal in previously used models. Killer whales are common around Iles Crozet during summer (Guinet 1992), but they feed mainly on penguins and elephant seals at this location. Although the risk of predation on fur seals can be high in some populations (Boveng et al. 1998), it is probably low at Ile de la Possession, because no females identified at La Mare aux Elephants were lost while nursing a pup, nor were there visible wounds or scars that could have been inflicted by a predator. Nonetheless, studies to determine whether predation risk differentially affects foraging behaviour of sympatric fur seals at Ile de la Possession may provide further insights into the mechanisms driving the observed differences in foraging behaviour.

Closely related to predation risk, the nutritional status of fur seals could also potentially influence foraging behaviour because animals may trade off energy gain with safety from predators (Nonacs 2001, Wirsing et al. 2008). Direct measurements of fat, the primary form of energy storage in pinnipeds, are not available for our study animals. However, a concurrent study showed that foraging trips at Ile de La Possession were among the briefest reported for each species at comparable stages of lactation (Luque et al. 2007a). Furthermore, the large proportion of overnight foraging trips, the relative proximity (50 to $100 \mathrm{~km}$ ) of foraging grounds (Bailleul et al. 2005), and the relatively fast growth rates of pups compared to other populations of both species, suggest that neither of them were under nutritional stress. Differences in energy stores between species under such conditions are expected to have minor influence on foraging behaviour (Houston et al. 1993, Nonacs 2001).

\section{Behavioural versus physiological differences}

We found conflicting evidence on the level of activity of fur seals, measured as the mean speed and absolute number of meters that fur seals swam during the bottom phase of dives. The former showed no differences between fur seal species, but the latter showed higher activity levels in SFS females. Speed is typically measured by counting the number of revolutions per unit time taken by a turbine on TDRs. These turbines are 
susceptible to clogging by debris (Shepard et al. 2008), rendering speed measurements for any given dive phase not as regular as depth measurements, despite similar sampling frequency. Consequently, fewer speed measurements were available for our comparisons of mean speed during any given dive phase, reducing the power of statistical comparisons relative to tests based on depth measurements. The vertical meters that fur seals swam per unit time during the bottom phase of dives may, thus, be a better index of activity level, and our results suggest that SFS females were more active during this phase. Despite the behavioural difference during the bottom phase of dives, SFS females showed increasing surface intervals following longer dives than AFS females.

Our data suggest that an important physiological parameter, the ADL, is higher in SFS than in AFS females. This result was unexpected and counter-intuitive because maximum dive depth was highest for AFS females, which was expected to be associated with a higher ADL. Lactating AFS females required exponentially longer periods at the surface when dive duration exceeded $150 \mathrm{~s}$, while SFS females responded similarly, but to dives exceeding $250 \mathrm{~s}$ in duration. Consistent with these observations, SFS dived deeper and for longer periods of time, on average. ADL calculations based on direct measurements of oxygen stores and diving metabolic rates of fur seals from South Georgia (Costa et al. 2004) yielded a value (96 s) that is much lower than that reported here, especially considering the larger body mass of individuals in that study. However, our data cannot readily be compared with direct measurements of ADL, and factors such as exceeding the ADL during deep diving bouts could account for the difference. Unfortunately, similar data are not available for SFS females, but if the tendency of study individuals to exceed the ADL is similar, then the observed interspecific differences may parallel actual differences in ADL. Costa et al. (2004) suggest that the tendency of epipelagic feeders, such as AFS and SFS, to exceed the ADL are indeed expected to be similar and relatively low. Although direct measurements of diving metabolism are needed to corroborate our results, the differences illustrated in Fig. 4 are likely a result of actual differences in ADL.

\section{Inferring foraging patch characteristics}

Previous analyses suggest that AFS and SFS females from La Mare aux Elephants used similar foraging areas during the 2001 and 2002 breeding seasons (Bailleul et al. 2005). Dietary analyses (Luque et al. 2007a) indicate that these fur seals also feed on the same myctophid fish species, albeit in different propor- tions (Luque et al. 2007a). Similar results have been found in sympatric populations at Macquarie Island (Robinson et al. 2002). The present study suggests that differences in physiological constraints may have led to different temporal and vertical distribution of diving, despite similarities in horizontal distribution of foraging locations and prey species. Whereas AFS foraged between the surface and $20 \mathrm{~m}$ more frequently than SFS females, the latter foraged more extensively between 30 and $60 \mathrm{~m}$. Furthermore, both species reduced the frequency of foraging below $80 \mathrm{~m}$, although SFS females did so more rapidly, and dive bouts below $140 \mathrm{~m}$ were only observed in AFS females. Assuming that dive bouts reflect foraging in different prey patches, AFS females exploited more patches per night of foraging, and a larger fraction of patches close to the surface. It may have been more profitable for AFS females to rapidly move between different patches at shallow depths, thereby reducing mean dive time and, possibly, also the extent of anaerobic diving (Costa et al. 2004).

Similar segregation of foraging depth has been observed in macaroni Eudyptes chrysolophus and gentoo Pygoscelis papua penguins at Bird Island, South Georgia (Mori \& Boyd 2004b), showing some analogies in their response with that of the fur seals in this study. Macaroni penguins increased surface times in response to briefer dive durations (Fig. 1 in Mori \& Boyd 2004b), so their ADL is presumably lower than that of gentoo penguins. Macaroni penguins concentrated their foraging bouts at shallower depths compared to gentoo penguins, analogous to the higher frequencies of shallow bout depths observed in AFS compared to SFS females at La Mare aux Elephants. Although direct measurements of prey density and abundance at different depths were not available in the present study to validate an index of patch quality (IPQ; Mori \& Boyd 2004a), this index can be calculated with the available data to examine potential interspecific differences in foraging patch quality. According to Mori \& Boyd (2004a), energy intake rate $(G)$ can be expressed in terms of dive duration $(u)$ and travel time from/to the surface $(\tau)$ as $G=a \times(u-\tau)^{x} /(u+y(u))$, where $y(u)$ is surface time as a function of dive duration, obtained from Fig. 4, and $a$ is an energy conversion constant. If divers regulate dive duration so as to maximize the rate of energy intake, the IPQ for a dive can be determined by finding the value of $x$ that maximizes $G$. The IPQ thus calculated indicates that foraging patch quality is higher for AFS below $50 \mathrm{~m}$, suggesting that this species foraged more profitably deeper in the water column than SFS (Fig. 5). Concurrent measurements of the distribution and densities of different prey species and the foraging behaviour of their air-breathing predators are needed to evaluate this suggestion. 


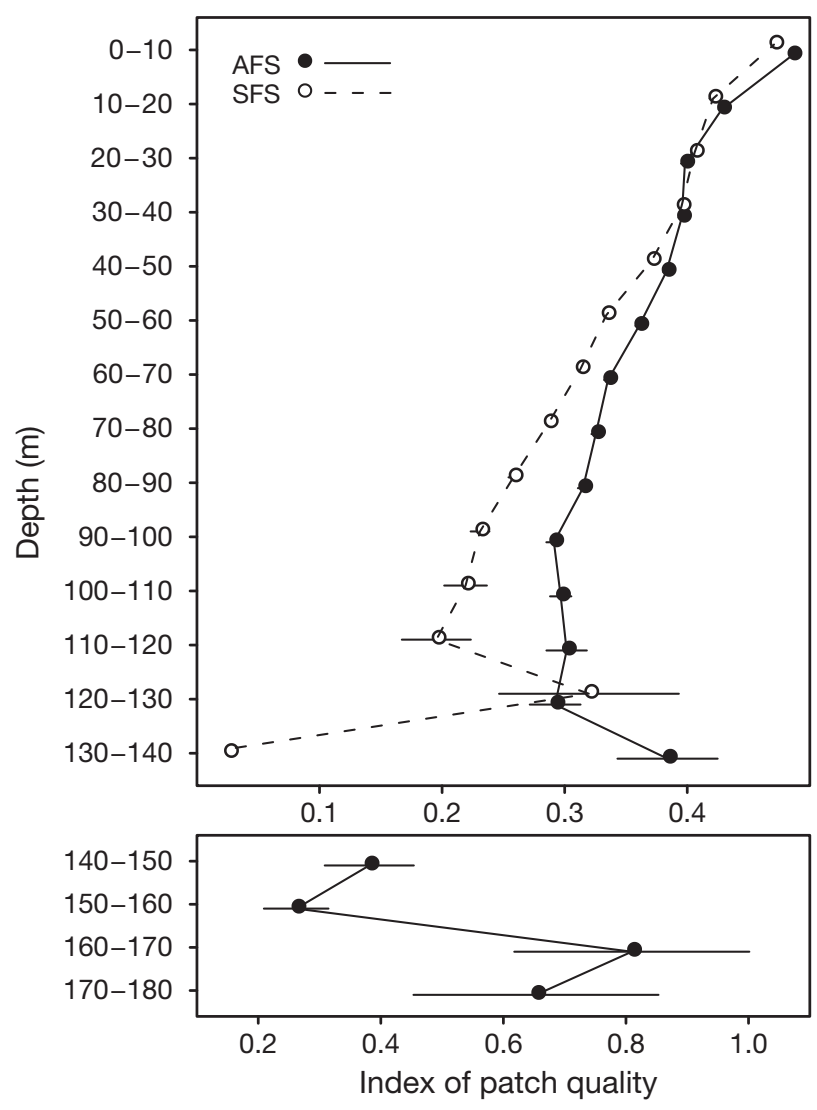

Fig. 5. Arctocephalus gazella (AFS), A. tropicalis (SFS). Lactating Antarctic fur seal (AFS) females encounter foraging patches with higher index of patch quality (IPQ) than subantarctic fur seal (SFS) females at depths between 50 and $100 \mathrm{~m}$. They also exploit patches at depths $>140 \mathrm{~m}$, which are not used by SFS and where variation in IPQ is large. Data for each species were shifted in opposite directions by a small amount on the vertical axis (within the same depth class) to avoid overlap of points. Values are means \pm SE

Since the IPQ may be similar between species at shallow depths, why did SFS females not exploit patches at these depths as extensively as AFS females did, despite their higher ADL? Conversely, why did AFS females exploit deep patches more extensively than their congenerics, despite their lower ADL?

Optimal diving models (Mori 1998a,b) predict that divers with higher ADLs should have deeper optimal foraging depth, for any given prey patch quality. These models also predict that anaerobic diving is favourable when the prey patch is deep and of high quality (Mori 1998b). SFS females may thus require higher prey patch qualities near the surface to dive optimally. Factors such as swimming mechanics (Fish et al. 2003, Sato et al. 2007), and searching and/or travelling behaviour (Hindell et al. 2002) may explain why these favourable conditions were encountered less frequently above 20 and below $80 \mathrm{~m}$ by SFS compared to
AFS females. For instance, both young and adult AFS and SFS show significant differences in fore- and hindflipper size and shape (Bester \& Wilkinson 1989, Luque et al. 2007b). Supporting this conjecture, AFS females showed a larger proportion of isolated dives, suggesting that they searched for foraging patches more extensively. Interestingly, activity during the bottom foraging phase of dives was higher in SFS, although their behavioural ADL was higher. This suggests that differences in the relationship between surface interval and dive duration were a result of physiological, rather than behavioural, constraints. Otherwise, the behavioural ADL would have been lower in SFS. Further studies comparing foraging efficiency between these species should help explain this pattern.

Indeed, the briefer bout durations and postbout intervals, and the larger proportion of exploratory dives observed in AFS females, may allow them to sample prey patches near the surface more frequently, and provide better knowledge of foraging conditions in this part of the habitat (Charnov 1976). Dive traces from AFS females at La Mare aux Elephants display stronger diel changes in dive depth than those from SFS females (Appendix 1), so that AFS find their deepest foraging patches during the crepuscular vertical migration of their myctophid prey (Boyd et al. 1994, McCafferty et al. 1998, Luque et al. 2007a). Some myctophid fish species of this sector of the Southern Ocean are known to form denser patches at their deep daytime depth locations than during the night (Bost et al. 2002), when they are patchily distributed near the surface. AFS, and to a much lesser extent SFS, may profit from the more densely aggregated fish during dawn and dusk, as they shift between these depth locations and spatial organizations. Our observations, therefore, suggest that AFS females spent a larger fraction of foraging time in patches of higher quality, particularly after dusk and before dawn, both during OFTs and LFTs, even when costs may have been higher due to deep (Luque et al. 2007a), anaerobic diving.

To conclude, we have shown interspecific differences in the temporal distribution of diving and foraging depth between sympatric AFS and SFS, which are consistent with the hypothesis that lactation pressure is higher in AFS. The larger number of bouts, briefer postbout interval, and higher propensity to dive anaerobically in AFS, suggest more effort spent at foraging. Furthermore, AFS appeared to concentrate that effort in prey patches of higher quality. These differences were associated with lower ADL, briefer lactation, and higher pup energy demands in AFS. Gentry et al. (1986) hypothesized that a suite of fur seal life history traits, including foraging behaviour and lactation duration, follow a latitudinal gradient, ultimately being determined by environmental seasonality and predictabil- 
ity. Additional information from species inhabiting temperate latitudes indicate that prey ecology may be more important than latitude in influencing foraging and maternal behaviours (Francis et al. 1998). Moreover, our results suggest that intrinsic differences in physiological constraints and lactational demands play important roles in determining the fine-scale foraging behaviour in sympatric populations of some species, despite similarities in diet and prey ecology.

Acknowledgements. We thank the Institut Polaire Français Paul Emile Victor (IPEV), the Draney-Anderson Foundation, and a graduate student fellowship (to S.P.L.) from the Biology Department of Memorial University for financial and logistic support. We also thank the members of the 39th and 40th missions to Ile de la Possession who participated in many aspects of field work, and several anonymous reviewers for valuable criticisms of the manuscript. Research was conducted in accordance with the guidelines set by IPEV for the Terres Australes et Antarctiques Françaises (French Antarctic and Austral Territories).

\section{LITERATURE CITED}

Arnould JPY (1997) Lactation and the cost of pup-rearing in Antarctic fur seals. Mar Mamm Sci 13:516-526

Arnould JPY, Luque SP, Guinet C, Costa DP, Kingston J, Shaffer SA (2003) The comparative energetics and growth strategies of sympatric Antarctic and subantarctic fur seal pups at Îles Crozet. J Exp Biol 206:4497-4506

Baayen RH, Davidson DJ, Bates DM (2008) Mixed-effects modeling with crossed random effects for subjects and items. J Mem Lang 53:390-412

Bailleul F, Luque SP, Dubroca L, Arnould JPY, Guinet C (2005) Differences in foraging strategy and maternal behaviour between two sympatric fur seal species at the Crozet Islands. Mar Ecol Prog Ser 293:273-282

Ballance LT, Pitman RL, Reilly SB (1997) Seabird community structure along a productivity gradient: importance of competition and energetic constraint. Ecology 78: 1502-1518

Bates D, Maechler M, Dai B (2008) Ime 4: linear mixed-effects models using S4 classes. R package version 0.999375-27. http://Ime4.r-forge.r-project.org

$>$ Beck CA, Bowen WD, Iverson SJ (2000) Seasonal changes in buoyancy and diving behaviour of adult grey seals. J Exp Biol 203:2323-2330

Bester MN, Bartlett PA (1990) Attendance behaviour of Antarctic and subantarctic fur seal females at Marion Island. Antarct Sci 2:309-312

Bester MN, Wilkinson IS (1989) Field identification of Antarctic and subantarctic fur seal pups. S Afr J Wildl Res 19: $140-144$

Birch LC (1957) The meanings of competition. Am Nat 91: $5-18$

Bonner N (1999) Seals and sea lions of the world. Blandford, London

Bost CA, Zorn T, Le Maho Y, Duhamel G (2002) Feeding of diving predators and diel vertical migration of prey: king penguins' diet versus trawl sampling at Kerguelen Islands. Mar Ecol Prog Ser 227:51-61

Boveng PL, Hiruki LM, Schwartz MK, Bengtson JL (1998) Population growth of Antarctic fur seals: limitation by a top predator, the leopard seal? Ecology 79:2863-2877

Boyd IL, Arnould JPY, Barton T, Croxall JP (1994) Foraging behaviour of Antarctic fur seals during periods of contrasting prey abundance. J Anim Ecol 63:703-713

> Caraco T (1980) On foraging time allocation in a stochastic environment. Ecology 61:119-128

Charnov EL (1976) Optimal foraging, the marginal value theorem. Theor Popul Biol 9:129-136

Costa DP, Williams TM (1999) Marine mammal energetics. In: Reynolds III JE (ed) Biology of marine mammals. Smithsonian Institution Press, Washington, DC, p 176-217

Costa DP, Kuhn CE, Weise MJ, Shaffer SA, Arnould JPY (2004) When does physiology limit the foraging behaviour of freely diving mammals? Int Congr Ser 1275: 359-366

Cowlishaw G (1999) Ecological and social determinants of spacing behaviour in desert baboon groups. Behav Ecol Sociobiol 45:67-77

Ferreira SM, Bester MN (1999) Chemical immobilization, physical restraint, and stomach lavaging of fur seals (Arctocephalus spp.) at Marion Island. S Afr J Wildl Res 29: 55-61

Fish FE, Hurley J, Costa DP (2003) Maneuverability by the sea lion Zalophus californianus: turning performance of an unstable body design. J Exp Biol 206:667-674

Francis J, Boness D, Ochoa-Acuña H (1998) A protracted foraging and attendance cycle in female Juan Fernández fur seals. Mar Mamm Sci 14:552-574

> Frid A, Heithaus MR, Dill LM (2007) Dangerous dive cycles and the proverbial ostrich. Oikos 116:893-902

Gentry RL, Kooyman GL (1986) Methods of dive analysis. In: Gentry RL, Kooyman GL (eds) Fur seals: maternal strategies on land and at sea. Princeton University Press, Princeton, NJ, p 28-40

Gentry RL, Costa DP, Croxall JP, David JHM and others (1986) Synthesis and conclusions. In: Gentry RL, Kooyman GL (eds) Fur seals: maternal strategies on land and at sea. Princeton University Press, Princeton, NJ, p 220-264

- Georges JY, Guinet C (2000) Early mortality and perinatal growth in the subantarctic fur seal (Arctocephalus tropicalis) on Amsterdam Island. J Zool (Lond) 251:277-287

Goldsworthy SD, Hindell MA, Crowley HM (1997) Diet and diving behaviour of sympatric fur seals Arctocephalus gazella and A. tropicalis at Macquarie Island. In: Hindell MA, Kemper C (eds) Marine mammal research in the Southern hemisphere: status, ecology and medicine. Surrey Beatty \& Sons, Chipping Norton, NSW, p 151-163

Guinet C (1992) Comportement de chasse des orques (Orcinus orca) autour des iles Crozet. Can J Zool 70:1656-1667

> Halsey LG, Butler PJ, Blackburn TM (2006) A phylogenetic analysis of the allometry of diving. Am Nat 167:276-287

> Heithaus MR, Frid A (2003) Optimal diving under the risk of predation. J Theor Biol 223:79-92

> Hindell MA, Harcourt R, Waas JR, Thompson D (2002) Finescale three-dimensional spatial use by diving, lactating female Weddell seals Leptonychotes weddellii. Mar Ecol Prog Ser 242:275-284

Hooker SK, Boyd IL, Jessopp M, Cox O, Blackwell J, Boveng P, Bengston JL (2002) Monitoring the prey-field of marine predators: combining digital imaging with datalogging tags. Mar Mamm Sci 18:680-697

Houston AI, Carbone C (1992) The optimal allocation of time during the diving cycle. Behav Ecol 3:255-265

Houston AI, McNamara JM, Hutchinson JMC (1993) General results concerning the trade-off between gaining energy and avoiding predation. Philos Trans R Soc Lond B Biol Sci 341:375-397

Hull CL (2000) Comparative diving behaviour and segregation of the marine habitat by breeding Royal penguins, 
Eudyptes schlegeli, and eastern Rockhopper penguins, Eudyptes chrysocome filholi, at Macquarie Island. Can J Zool 78:333-345

Kato A, Watanuki Y, Nishiumi I, Kuroki M, Shaughnessy P, Naito Y (2000) Variation in foraging and parental behaviour of king cormorants. Auk 117:718-730

Klages NTW, Bester MN (1998) Fish prey of fur seals Arctocephalus spp. at subantarctic Marion Island. Mar Biol (Berl) 131:559-566

Kooyman GL (1989) Diverse divers: physiology and behavior. Springer-Verlag, Berlin

> Langton SD, Collett D, Sibly RM (1995) Splitting behaviour into bouts; a maximum likelihood approach. Behaviour 132: 781-799

Luque SP (2007) Diving behaviour analysis in R. R News 7: 8-14

Luque SP, Guinet C (2007) A maximum likelihood approach for identifying dive bouts improves accuracy, precision, and objectivity. Behaviour 144:1315-1332

Luque SP, Arnould JPY, Miller EH, Cherel Y, Guinet C (2007a) Foraging behaviour of sympatric Antarctic and subantarctic fur seals: does their contrasting duration of lactation make a difference? Mar Biol 152: 213-224

Luque SP, Miller EH, Arnould JPY, Chambellant M, Guinet C (2007b) Ontogeny of body size and shape of Antarctic and subantarctic fur seals. Can J Zool 85:1275-1285

MacArthur R, Levins R (1964) Competition, habitat selection, and character displacement in a patchy environment. Proc Natl Acad Sci USA 51:1207-1210

McCafferty DJ, Boyd IL, Walker TR, Taylor RI (1998) Foraging responses of Antarctic fur seals to changes in the marine environment. Mar Ecol Prog Ser 166:285-299

Mehlum F, Watanuki Y, Takahashi A (2001) Diving behaviour and foraging habitats of Brünnich's guillemots (Uria lomvia) breeding in the High-Arctic. J Zool (Lond) 255: 413-423

Milinski M, Parker GA (1991) Competition for resources. In: Krebs JR, Davies NB (eds) Behavioural ecology: an evolutionary approach. Blackwell Scientific Publications, Oxford, p 137-168

Mori Y (1998a) Optimal choice of foraging depth in divers. J Zool (Lond) 245:279-283

Mori Y (1998b) The optimal patch use in divers: optimal time budget and the number of dive cycles during the bout. J Theor Biol 190:187-199

Mori Y (2002) Optimal diving behaviour for foraging in relation to body size. J Evol Biol 15:269-276

Mori Y, Boyd IL (2004a) The behavioral basis for nonlinear functional responses and optimal foraging in Antarctic fur seals. Ecology 85:398-410

Mori Y, Boyd IL (2004b) Segregation of foraging between two sympatric penguin species: does rate maximization make the difference? Mar Ecol Prog Ser 275:241-249

Mori Y, Yoda K, Sato K (2001) Defining dive bouts using a sequential differences analysis. Behaviour 138:1451-1466

Editorial responsibility: Hans Heinrich Janssen, Oldendorf/Luhe, Germany
Mori Y, Takahashi A, Mehlum F, Watanuki Y (2002) An application of optimal diving models to diving behaviour of Brünnich's guillemots. Anim Behav 64:739-745

Mori Y, Watanabe Y, Mitani Y, Sato K, Cameron MF, Naito Y (2005) A comparison of prey richness estimates for Weddell seals using diving profiles and image data. Mar Ecol Prog Ser 295:257-263

Nonacs P (2001) State dependent behavior and the marginal value theorem. Behav Ecol 12:71-83

Pinheiro JC, Bates DM (2000) Mixed-effects models in S and S-PLUS. Springer-Verlag, New York

R Development Core Team (2007) R: a language and environment for statistical computing. R Foundation for Statistical Computing, Vienna, www.R-project.org

Ridgway SH, Harrison RJ (eds) (1981) Handbook of marine mammals, I. The walrus, sea lions, fur seals and sea otters. Academic Press, San Diego, CA

Robinson SA, Goldsworthy SD, van den Hoff J, Hindell MA (2002) The foraging ecology of two sympatric fur seal species, Arctocephalus gazella and Arctocephalus tropicalis, at Macquarie Island during the austral summer. Mar Freshw Res 53:1071-1082

Sæther BE, Gordon IJ (1994) The adaptive significance of reproductive strategies in ungulates. Proc R Soc Lond B Biol Sci 256:263-268

Sato K, Watanuki Y, Takahashi A, Miller PJO and others (2007) Stroke frequency, but not swimming speed, is related to body size in free-ranging seabirds, pinnipeds, and cetaceans. Proc R Soc Lond B Biol Sci 274:471-477

Schoener TW (1974) Resource partitioning in ecological communities. Science 185:27-39

> Shepard ELC, Wilson RP, Liebsch N, Quintana F, Gómez Laich A, Lucke K (2008) Flexible paddle sheds new light on speed: a novel method for the remote measurement of swim speed in aquatic animals. Endang Species Res 4: $157-164$

Sibly RM, Nott HMR, Fletcher DJ (1990) Splitting behaviour into bouts. Anim Behav 39:63-69

> Stamps JA (1983) The relationship between ontogenetic habitat shifts, competition and predator avoidance in a juvenile lizard (Anolis aeneus). Behav Ecol Sociobiol 12:19-33

Thompson D, Fedak MA (2001) How long should a dive last? A simple model of foraging decisions by breath-hold divers in a patchy environment. Anim Behav 61:287-296

Tilman D (1987) The importance of the mechanisms of interspecific competition. Am Nat 129:769-774

Trillmich F, Kooyman GL (1986) Attendance behavior of Galápagos fur seals. In: Gentry RL, Kooyman GL (eds) Fur seals: maternal strategies on land and at sea. Princeton University Press, Princeton, NJ, p 168-187

Wirsing AJ, Heithaus MR, Frid A, Dill LM (2008) Seascapes of fear: evaluating sublethal predator effects experienced and generated by marine mammals. Mar Mamm Sci 24: $1-15$

Submitted: May 6, 2008; Accepted: August 13, 2008 Proofs received from author(s): November 21, 2008 